Revue internationale P.M.E.

Économie et gestion de la petite et moyenne entreprise

Revure

internationale

PME

\title{
La planification dans les PME : une synthèse de résultats empiriques \\ Conclusions et recommandations
}

\section{Gérald d'Amboise et Athanase Bakanibona}

Volume 3, numéro 2, 1990

URI : https://id.erudit.org/iderudit/1007975ar

DOI : https://doi.org/10.7202/1007975ar

Aller au sommaire du numéro

Éditeur(s)

Presses de l’Université du Québec

ISSN

0776-5436 (imprimé)

1918-9699 (numérique)

Découvrir la revue

Citer cet article

d'Amboise, G. \& Bakanibona, A. (1990). La planification dans les PME : une synthèse de résultats empiriques : conclusions et recommandations. Revue internationale P.M.E., 3(2), 147-166. https://doi.org/10.7202/1007975ar
Résumé de l'article

Cet article est basé sur une revue sélective d'études empiriques axées sur la problématique de la planification dans les PME. Les résultats d'études récentes ayant particulièrement essayé d'établir la relation entre les activités de planification et la performance dans les petites et moyennes entreprises sont présentés et discutés. Quelques études traitent de la planification en général ; certaines font la distinction appropriée entre les activités de planification stratégique et celles de nature opérationnelle. Les regroupements effectués ici tiennent compte de ces précisions qui favorisent une meilleure compréhension du phénomène dans les PME. Quelques conclusions synthétisent les résultats relatifs à l'utilité des diverses formes de planification dans les firmes de moins grande envergure. Les auteurs offrent également quelques suggestions dans le but de faire progresser ce domaine de recherche. 


\title{
La planification dans les PME : une synthèse de résultats empiriques Conclusions et recommandations
}

\author{
Gérald d'AMBOISE* \\ Université Laval \\ Athanase BAKANIBONA* \\ Université Laval
}

\begin{abstract}
RÉSUMÉ
Cet article est basé sur une revue sélective d'études empiriques axées sur la problématique de la planification dans les PME. Les résultats d'études récentes ayant particulièrement essayé d'établir la relation entre les activités de planification et la performance dans les petites et moyennes entreprises sont présentés et discutés. Quelques études traitent de la planification en général; certaines font la distinction appropriée entre les activités de planification stratégique et celles de nature opérationnelle. Les regroupements effectués ici tiennent compte de ces précisions qui favorisent une meilleure compréhension du phénomène dans les PME. Quelques conclusions synthétisent les résultats relatifs àl'utilité des diverses formes de planification dans les firmes de moins grande envergure. Les auteurs offrent également quelques suggestions dans le but de faire progresser ce domaine de recherche.
\end{abstract}

* Gérald d'Amboise est professeur de management à la Faculté des Sciences de l'administration de l'Université Laval. Il a réalisé et publié plusieurs études sur les pratiques particulières de gestion des petites et moyennes entreprises. Athanase Bakanibona est originaire du Burundi. Au moment de la préparation de cet article, il était étudiant à la maîtrise en administration des affaires à l'Université Laval. Adresse: Faculté des Sciences de l'administration, Université Laval, Cité universitaire, Ste-Foy, Québec, G1K 7P4. Premier manuscrit reçu: février 1990. 


\begin{abstract}
This article is based on a selective review of empirical studies dealing with the issue of planning in small and medium-sized businesses. The results of recent studies that specifically tried to establish the relationship between planning activities and performance in small and mediumsized businesses are presented and discussed. A few studies deal with planning in general; others more appropriately distinguish between strategic planning and operational planning activities. The grouping presented here takes these specifications into account since they facilitate the comprehension of the phenomenon in small and medium-sized businesses. A few conclusions summarize the results with respect to the usefulness of various forms of planning in smaller firms. The authors also provide a few suggestions in order to contribute to the progress of research in this field.
\end{abstract}

\title{
RESUMEN
}

Este articulo esta fundado sobre una revision selectiva de estudios empiricos que tratan de la problematica de la planificacion en las empresas pequenas y medianas. También estan presentados y discutidos los resultados de estudios recientes cuyo objetivo consistia en establecer la relacion entre las actividades de planificacion y el rendimiento en las empresas pequenas y medianas. Algunos estudios tratan de la planificacion en general; otros hacen la distincion apropiada entre la planificacion estratégica y la de tipo operacional. En los agrupamientos hechos aqui, tomamos esas especificaciones en cuenta ya que favorecen una mejor comprension del fenomeno en las empresas pequenas y medianas. Algunas conclusiones sitetizan los resultados relacionados con la utilidad de varias formas de planificacion en las empresas mas pequenas. Los autores ofrecen también al gunas sugerencias con el fin de hacer progresar las investigaciones en este ambito. 


\section{Introduction}

Est-il bénéfique de planifier pour une petite ou moyenne entreprise (PME) ? La planification a-t-elle un lien avec la performance ? Quel est l'effet de la formalisation ou de la non-formalisation de la planification sur la performance?

De telles interrogations ne manquent certes pas d'intérêt comme le prouve l'étendue de la littérature disponible sur le sujet de la planification. Malheureusement la plupart des chercheurs ont clairement privilégié la grande entreprise dans leurs travaux; ils sont de plus en plus obligés d'admettre qu'il n'est cependant pas toujours réaliste de transférer à la PME les conclusions de leurs études. Aussi, depuis quelques années, le besoin de mieux comprendre le rôle de la planification, particulièrement dans les PME, devient-il de plus en plus évident? Certains chercheurs orientent donc leurs analyses en planification vers les PME. che $^{1}$ :

Quatre raisons au moins militent en faveur de cette orientation de recher-

1) Les ressources des PME sont relativement limitées.

2) Leurs possibilités de choix stratégiques sont moins nombreuses.

3) Leur contribution à l'économie est très importante (ex. : leur nombre, part de l'emploi, rôle dans plusieurs secteurs d'activités, etc.).

4) Elles constituent actuellement en particulier une importante source d'emplois et d'innovation'.

Un des outils préalables à des recherches potentielles plus poussées est certainement une synthèse de la littérature déjà existante sur la planification dans les PME. R.B. Robinson et J.A. Pearce ont déjà produit une synthèse de ce genre pour les études faites avant 1984.

L'objectif de la présente initiative est de faire une revue sélective d'études empiriques axées sur la même problématique de la planification dans les PME et publiées depuis 1983. Les conclusions émergentes de plusieurs études réalisées auparavant seront d'abord rappelées; le bilan de 1984 des auteurs Robinson et Pearce servira de référence principale à cet effet. Seront ensuite présentés et discutés les résultats d'études empiriques plus récentes ayant particulièrement essayé d'établir la relation entre les activités de planification et la performance dans les petites et moyennes entreprises. Quelques conclusions générales tenteront de faire le point sur le sujet de la planification dans les PME. Certaines recommandations susceptibles de faire progresser ce domaine de recherche seront également formulées.

1 Voir R.B. Robinson et J.A. Pearce (1984).

2 Voir par exemple : P.A. Julien et M. Marchesnay (1988) ou G. d'Amboise (1989: 47-64). Tous les relevés démontrent que les PME occupent une place importante dans les pays industrialisés. Leur part est encore plus importante dans les économies de plusieurs pays moins avancés. 


\section{Le bilan de R.B. Robinson et J.A. Pearce}

En 1984, R.B. Robinson et J.A. Pearce ont comblé un vide en faisant une analyse de la littérature antérieure portant sur la planification dans les PME. Déjà, il ressort de leur analyse que la planification est importante pour les PME et que son existence (ou son absence) conditionne leur survie (Robinson et Pearce, 1984:128).

Certaines recherches faites avant 1984 observent des pratiques qui prouvent l'existence de la planification. Toutefois, elles décrivent la planification dans les PME comme incomplète, non-structurée, irrégulière, sporadique, plutôt réactive et informelle (Still, 1974; Shuman, 1975; Sexton et Dable, 1976).

Une des principales conclusions de R.B. Robinson et J.A. Pearce est que le processus de planification préconisé pour les PME est jugé trop complexe et trop sophistiqué. Le temps qu'il requiert limite beaucoup son utilisation. Déjà certaines des études incluses dans leur analyse préconisaient un processus court (moins de 2 ans) et plutôt informel. Le concours de plusieurs participants (y compris des personnes externes à l'entreprise) y était également suggéré afin de favoriser la créativité (Robinson et Pearce, 1984:129).

Quoique sujette à controverses, une relation positive entre la planification et la performance a été observée par Trow (1961), Najjar (1966) et Robinson (1979). De même, Wyant (1977) et Mayer et Goldstein (1961) ont conclu que l'absence de planification est une des causes importantes de faillites dans les PME. D'autres auteurs vont plus loin et concluent qu'il existe un lien positif entre le niveau de sophistication de la planification et la performance (Chicha, 1979; Bracker, 1982), puis entre l'utilisation de consultants et la performance (Robinson, 1980 et 1982).

A l'opposé, une étude sur les banques a abouti à la conclusion que la grande formalisation de la planification (high formality) n'améliore pas la performance (Robinson et Pearce, 1983). Une autre recherche sur le commerce de détail et les usines de fabrication conclut que c'est la planification informelle qui améliore la performance (Robinson, 1980).

La principale conclusion du bilan de R.B. Robinson et J.A. Pearce est que la planification est importante pour les PME, car son existence ou son absence conditionne la survie des PME. Mais le fait que ce soit la planification formelle qui améliore la performance des PME (plutôt que la planification informelle) est soutenu par certains chercheurs et mis en doute par d'autres. Cette controverse se retrouve encore dans les études plus récentes comme on pourra l'observer dans ce nouveau recensement. 


\section{$3 \quad$ Les travaux récents}

Sous cette section est proposée une analyse sélective des recherches menées sur la planification dans la PME et publiées depuis 1984.

\subsection{Critères de sélection} suivants :

Cette analyse se limite aux seules études répondant strictement aux critères

- les résultats découlent de données empiriques ${ }^{3}$

- les échantillons contiennent des PME uniquement ${ }^{4}$

- on tente d'établir une relation entre la planification et la performance ${ }^{5}$

— l'étude doit avoir été publiée depuis 1983.

\subsection{Classification des travaux sélectionnés}

Compte tenu de ces critères, douze recherches ont été retenues et classées en 3 catégories :

Catégorie 1: recherches analysant la relation entre la planification stratégique et la performance : 8 recherches

Catégorie 2: recherches analysant la relation entre la planification opérationnelle et la performance : 3 recherches

Catégorie 3: recherches analysant la relation entre d'autres aspects de la planification et la performance : 1 recherche ${ }^{6}$.

On constatera que selon qu'elles se limitent à la seule existence de la planification ou qu'elles analysent son envergure (formalisation et sophistication de la planification), les recherches de la première catégorie ont été subdivisées en souscatégories (voir le tableau 1, p. 152).

3 Ce critère élimine les conclusions prescriptives de certaines études théoriques et sans base empirique évidente.

4 La définition de la PME varie d'un auteur à l'autre. Ce travail-ci a retenu les recherches qui qualifient explicitement les firmes de leur échantillon de «small business» ou de «PME». Quand la taille des firmes était donnée, on a utilisé la définition de G. d'Amboise (1989:25), soit moins de 500 employés et moins de 20 millions de dollars de ventes annuelles.

5 Les auteurs de ce travail considèrent les analyses basées sur les résultats d'associations statistiques avec une mesure de performance comme étant plus révélatrices de l'utilité de la planification.

6 Les revues recensées traitent prioritairement de PME ou de planification. Elles se limitent à celles publiées soit au Canada soit aux États-Unis. Les termes utilisés par les divers auteurs pour identifier les variables sous observation ont été strictement retenus. Ainsi, l'auteur qui utilise le terme «planification stratégique» voit sa publication classée dans la catégorie 1 du présent tableau. 
Tab. 1 Classification des travaux récents

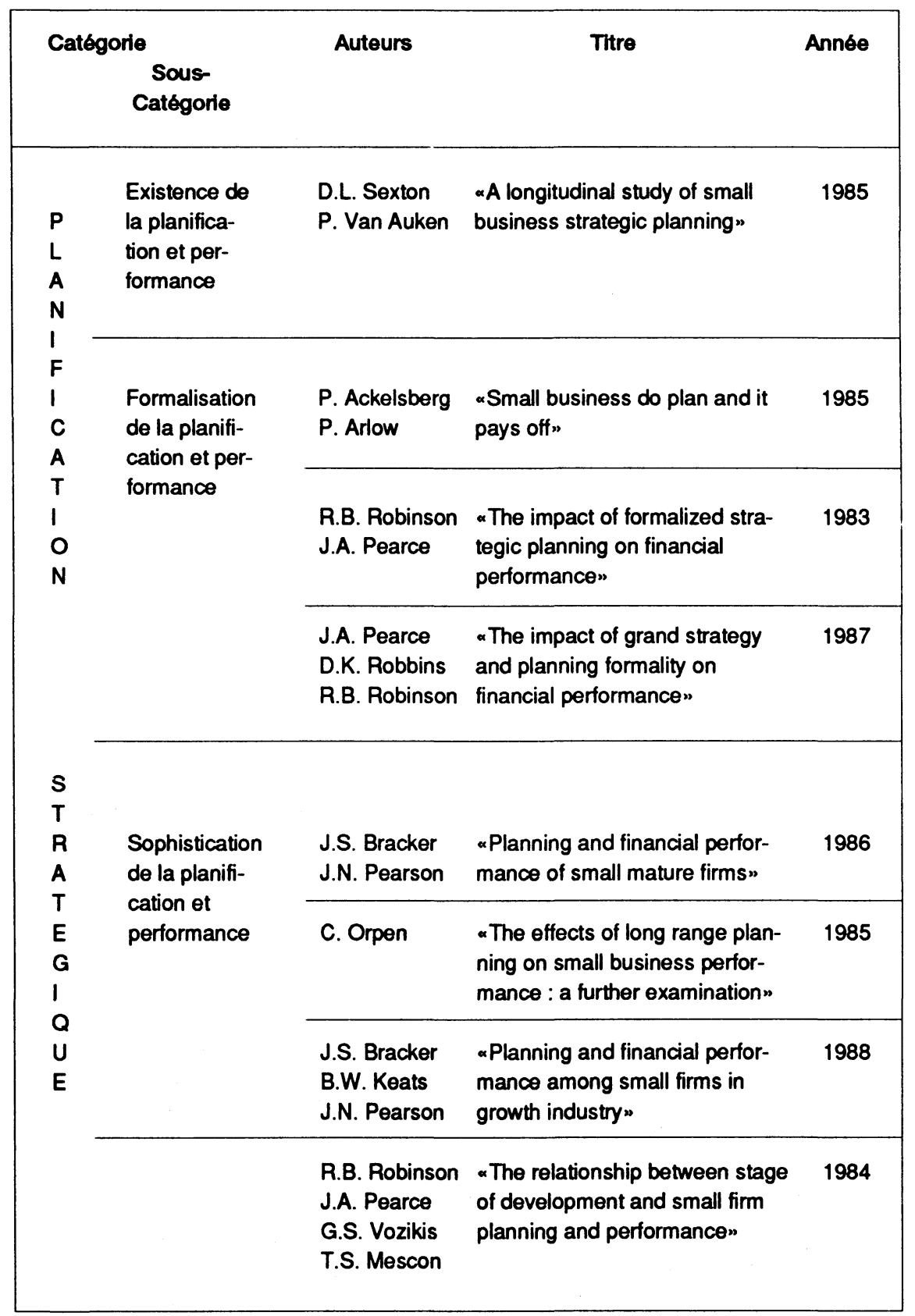




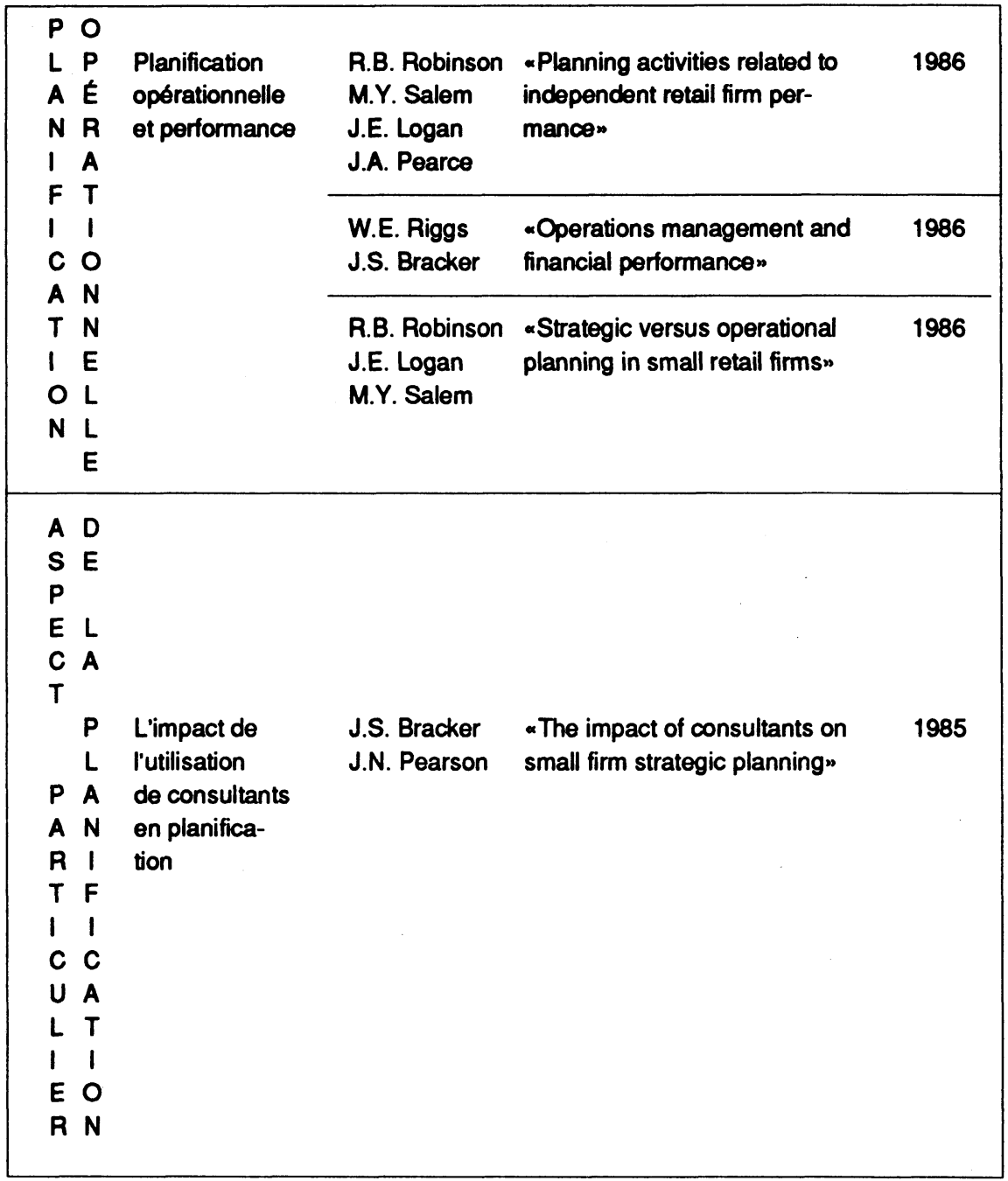

\subsection{Résultats des travaux retenus}

\subsubsection{L'existence de la planification dans les PME}

Les travaux retenus pour les fins de cette analyse montrent bien l'existence d'une certaine planification dans les entreprises étudiées. Ainsi, dans un échantillon de 81 PME du secteur du commerce de détail, R.B. Robinson, M.Y. Salem, J.E. Logan et J.A. Pearce (1986:22) observent qu'une partie prépondérante des firmes déclare s'occuper des activités suivantes «dans une large ou une très large mesure» : 
- la détermination du niveau de la quantité à commander (69\% des répondants)

- le calcul du point-mort des ventes (60\% des répondants)

- la comparaison entre les ventes prévues et celles réalisées (54\% des répondants).

Dans un échantillon de $555 \mathrm{PME}$ du secteur du nettoyage à sec, W.E. Riggs et J.S. Bracker (1986:21) constatent que sur 183 répondants, 74 font de la prévision de ventes et 38 de la planification globale.

De même, J.S. Bracker et J.N. Pearson (1985:27) observent sur un échantillon de 188 firmes de nettoyage à sec que 148 d'entre elles utilisent même des consultants en planification.

Par contre, R.B. Robinson, J.E. Logan et M.Y. Salem (1986:15) constatent que $85 \%$ de leur échantillon de 81 épiceries ne font pas systématiquement de la planification stratégique. Cela signifie que seul les $15 \%$ restants le fait. Ces constatations correspondent à celles de D.L. Sexton et P. Van Auken (1985:9 et 10) faites lors de leurs travaux en 1981 auprès de 357 PME de divers secteurs d'activités. Alors que bon nombre de dirigeants d'entreprises estimaient leurs ventes annuelles, un très faible pourcentage d'entre eux effectuaient une véritable planification stratégique. Ces auteurs ont déterminé que $18 \%$ seulement de ces dirigeants s'adonnaient à une planification stratégique globale.

Tels sont déjà quelques-uns des résultats des recherches sélectionnées. La proportion des entreprises qui planifient varie d'une étude à l'autre; les activités de planification sont en général assez limitées. Comme on doit souvent l'admettre, le choix de tels échantillons est rarement fait sur une base vraiment aléatoire. Les résultats ne proviennent que des répondants qui ont bien voulu collaborer. Ils ne reflètent certainement pas le véritable degré d'utilisation de la planification dans les PME. Cependant, ces résultats peuvent servir de bonne indication surtout lorsque l'échantillon provient d'un seul secteur d'activités.

\subsubsection{Relation entre planification et performance}

Deux niveaux de planification sont généralement distingués dans la littérature : la planification stratégique et la planification opérationnelle. La même distinction se retrouve dans les études axées sur la relation entre la planification et la performance.

\subsubsection{Planification stratégique et performance}

Différents aspects de la planification stratégique peuvent influencer le niveau de performance d'une entreprise (l'existence de la planification stratégique, sa formalisation, son niveau de sophistication). L'impact propre à chacun d'entre eux est discuté séparément par certaines des études.

\subsection{Existence de la planification stratégique et performance}

La plupart des études révèlent que les dirigeants de PME font peu de planification; ces activités sont souvent parcellaires et sporadiques. Les entreprises 
qui bénéficient d'une certaine planification connaissent toutefois plus de succès que les autres. Quelques auteurs ont effectivement établi une relation entre l'existence d'activités de planification dans des PME et leur niveau de performance. $R$. Ackelsberg et P. Arlow (1985:62) ont trouvé par exemple que les firmes qui planifient voient leurs ventes et leurs profits augmenter proportionnellement plus que celles qui ne le font pas. De leur côté, D.L. Sexton et P. Van Auken (p. 12 et 13) poursuivant leurs travaux antérieurs auprès du même groupe d'entreprises faisaient d'autres constatations. D'une part, les dirigeants qui ne s'adonnent pas à une planification assez globale ne le font pas davantage 2 ans plus tard. Selon eux, les dirigeants qui adoptent au départ une approche restreinte aux opérations du court terme s'en tiennent longtemps à cette dernière (p. 12). Ils rapportent d'autre part que 47 entreprises de l'échantillon original avaient subi la faillite. Une proportion de $20 \%$ de celles qui ne planifiaient pas au moment de la première rencontre avait disparu; un pourcentage moindre $(8 \%)$ de celles qui s'y adonnaient plus sérieusement avaient dû fermer leurs portes. Ils concluent que «strategic planning appears to help many firms to survive in a competitive market». (p. 13). L'utilité de la planification est plus adéquatement confirmée par les analyses plus précises qui seront maintenant rapportées.

\subsection{Formalisation de la planification et performance}

La relation entre la formalisation de la planification et la performance est très controversée dans la littérature. Certaines études affirment que la formalisation de la planification améliore la performance, mais cette idée est rejetée par les conclusions d'autres recherches.

Pour ne citer que quelques exemples, J.A. Pearce, R.B. Robinson et D.K. Robbins (1987:131 et 132) concluent à partir d'une étude sur un échantillon de 97 PME manufacturières que la formalisation de la planification apparaît comme systématiquement bénéfique pour les PME et qu'il existe une relation positive et significative entre la planification et la performance en matière de ventes.

Dans le même sens, C. Orpen (1985:19) constate sur un échantillon de 52 PME que les firmes les plus performantes utilisent plus la planification formelle que les moins performantes.

A l'opposé, R.B. Robinson et J.A. Pearce (1983:204) concluent que la formalisation de la planification ne conduit pas à une meilleure performance que la planification non-formelle. Leur échantillon était composé de 85 banques.

De même, R. Ackelsberg et P. Arlow (1985:62) constatent que dans le cas particulier des entreprises manufacturières contenues dans leur échantillon, les ventes sont inversement proportionnelles au niveau de formalisation de leurs activités de planification.

Cette divergence des résultats des études qui ont tenté d'analyser la relation entre la formalisation de la planification et la performance fait penser que la seule 
formalisation de la planification ne rend pas compte de la valeur de la planification. Etant donné le mode de gestion, la taille et la structure de beaucoup de PME, on peut se demander si une planification très formalisée est toujours nécessaire. Vite et tôt, la formalisation pourrait devenir excessive pour une entreprise d'envergure réduite. Déjà avant 1984, les recommandations de certains chercheurs ne préconisaient-elles pas un processus de planification plutôt informel pour les PME ? (Gasse, 1979; Robinson, 1980; Pearce et Robinson, 1983).

\subsection{Niveau de sophistication de la planification et performance}

Au-delà de la simple formalisation, le niveau de sophistication de la planification semble davantage révélateur'. Quelques auteurs l'ont approfondi en rapport avec la performance des PME qu'ils ont analysées ${ }^{8}$.

Plusieurs ont observé un lien significatif, par exemple, entre le niveau de sophistication générale de la planification et la performance financière (Bracker et Pearson, 1986:152; Bracker, Keats et Pearson, 1988:597).

D'autres ont préféré considérer un aspect particulier de la sophistication de la planification, à savoir l'intensité de la planification stratégique'. Ils constatent à partir d'un échantillon de 51 PME que l'intensité de la planification stratégique a une forte relation avec la performance (Robinson, Pearce, Vozikis et Mescon, 1984:51). Dans une étude basée sur 217 firmes du secteur de l'électronique, J.S. Bracker, B.W. Keats et J.N. Pearson (1988:597) relèvent que les PME utilisant une planification structurée ont une meilleure performance globale que les autres firmes.

Mais une des contributions les plus importantes en matière de planification revient à l'étude de C. Orpen qui, en 1985, établissait sur la base d'un échantillon de 52 PME que c'est moins le temps consacré que la qualité de la planification qui différencie les dirigeants de PME à rendement élevé de ceux des PME à rendement faible.

7 Voir conclusion similaire chez J.S. Bracker, B.W. Keats et J.N. Pearson (1988:599).

8 Le niveau de sophistication de la planification (planning sophistication) est estimé par le caractère plus ou moins structuré (contre intuitif) et plus ou moins stratégique (contre opérationnel) de la planification (Bracker et Pearson, 1986:507). Alors que la formalisation peut déjà tenir du simple fait de l'existence d'un plan écrit, la sophistication de la planification est plutôt reliée à la structure du processus, à la prise en compte de l'environnement, à l'horizon visé et à l'intégration de la planification dans les fonctions de l'entreprise.

9 Selon R.B. Robinson, J.N. Pearce, G.S. Vozikis et T.S. Mescon (1984:49), l'intensité de la planification stratégique s'analyse à travers:

- le niveau d'intégration de la planification stratégique dans les fonctions de la firme

- le nombre d'heures investies en planification stratégique

- le nombre de consultants participant à la planification stratégique. 


\subsection{L'effet de variables modératrices}

Un certain nombre de variables modératrices peuvent influer sur la relation entre la planification et la performance d'une PME. Parmi celles qui ont été analysées dans les travaux récents, on retrouve : l'expérience en planification, le stade de développement de l'entreprise, sa taille et le type d'entrepreneur.

\section{a) L'expérience en planification}

L'étude déjà citée, menée en 1986 sur 555 PME du secteur du nettoyage à sec indiquait qu'il existe un lien significatif entre l'expérience en planification et la performance financière (Bracker et Pearson, 1986:512). Plus nuancée a été la conclusion tirée deux années plus tard à partir d'une étude menée sur 217 PME du secteur de l'électronique. Les auteurs de cette étude ont observé qu'il n'y a pas de différence significative de performance due à l'écart d'expérience en planification. Mais les mêmes auteurs ont constaté que dans un groupe de PME de longue expérience en planification, la meilleure performance s'observe chez celles utilisant une planification stratégique structurée (Bracker, Keats et Pearson, 1988:597).

\section{b) Le stade de développement de l'entreprise}

L'étude de R.B. Robinson, J.N. Pearce, G.S. Vozikis et T.S. Mescon (1984) souligne que le niveau de performance des PME engagées en planification n'est pas reliéà leur stade de développement. Elle rejette aussi l'influence du stade de développement sur la relation entre la planification et la performance. Cette variable modératrice n'a cependant pas été analysée par d'autres chercheurs.

\section{c) La taille de l'entreprise}

La même étude basée sur 217 PME de l'électronique rejette l'existence d'un effet significatif de la taille des firmes sur la relation entre la planification et la performance (Bracker, Keats et Pearson, 1988:597). Néanmoins, l'étude reconnaît que les firmes les plus grandes ont une plus forte croissance du profit que les plus petites.

\section{d) Le type d'entrepreneur}

Seule la même étude de J.S. Bracker, B.W. Keats et J.N. Pearson (1988) a analysé l'effet de cette variable modératrice sur la relation entre la planification et la performance de l'entreprise. Elle se limite à signaler que les données permettent de «présumer» une forte relation entre les caractéristiques de l'entrepreneur et le niveau de sophistication de la planification dans sa firme.

En particulier, l'étude constate que parmi les firmes possédées et dirigées par des entrepreneurs «opportunistes $»^{10}$, la meilleure performance se retrouve chez

10 Cité par J.S. Bracker, B.W. Keats et J.N. Pearson (1988), N. Smith précise les caractéristiques propres à chacun des deux types d'entrepreneurs comme suit: 
La planification dans les PME : une synthèse de résultats empiriques,

celles utilisant une planification stratégique structurée. D'après l'analyse, une planification structurée se retrouve dans tout groupe de PME parvenues à la maturité, et propriétés d'entrepreneurs «opportunistes».

De toute cette analyse de la relation entre la planification stratégique et la performance découlent quelques constatations générales qui méritent d'être rappelées. D'une part la planification est utile pour les PME; d'autre part, la seule existence d'un plan écrit ne rend pas compte de la valeur de ce plan. Cette nuance peut être à la base de divergences de résultats d'analyses de la relation entre la formalisation de la planification et la performance. Par contre, le niveau de sophistication de la planification semble avoir plus d'effet sur la performance, surtout quand la structure du processus de planification et l'intégration de celle-ci dans les fonctions de l'entreprise sont assez avancées. Enfin, le bon rendement des entreprises est davantage conditionné par la qualité de la planification que par le temps qui lui est consacré.

\subsubsection{Planification opérationnelle et performance}

La planification opérationnelle est un aspect important pour un bon suivi des activités de l'entreprise. Ce volet de la planification conditionne la performance à court terme. Quelques auteurs ont explicitement abordé les activités de planification opérationnelle dans les PME qu'ils ont étudiées.

Ainsi, dans une étude conduite à ce sujet dans 81 épiceries et publiée en 1986, il a été constaté que 13 activités de planification opérationnelle expliquent $82 \%$ des variations du profit réalisé de 1981 à $1983 .{ }^{11}$ Parmi elles, les 6 activités ciaprès se sont particulièrement avérées positivement reliées au niveau du profit et à la performance perçue par le dirigeant :

- L'analyse des changements possibles dans le marché-cible

- La recherche et l'analyse de données sur les grands concurrents

- La prévision réaliste des ventes

- La prévision des flux mensuels de trésorerie

- L'établissement et la révision des coûts standards de main-d'oeuvre

«L'entrepreneur opportuniste (opportunistic entrepreneur) est stimulé par un assez vaste éventail culturel; il manifeste un niveau d'instruction et de formation élevé; il est socialement éveillé, flexible, confiant et orienté vers le futur. L'entrepreneur artisan (craftsman entrepreneur) démontre des caractéristiques opposées sur chacune des mêmes dimensions.»

11 G. Steiner (cité par R.B. Robinson, J.E. Logan et M.Y Salem, 1986) définit les concepts de eplanification opérationnelle» et de eplanification stratégique» comme suit:

«La planification opérationnelle (operational planning) correspond aux activités de planification détaillées qui permettent l'élaboration d'objectifs à court terme, de plans d'action et de procédures pour la conduite des opérations quotidiennes. La planification stratégique (strategic planning), au contraire, consiste à définir la mission d'une entreprise, déterminer ses objectifs à long terme et choisir les stratégies pour les atteindre.» 
- La révision périodique du niveau minimum d'inventaire pour chaque article important. ${ }^{12}$

Il en est de même pour l'étude (déjà mentionnée) sur 555 firmes de nettoyage à sec, laquelle a permis de constater que les PME qui font de la prévision de demande et de la planification globale ont une meilleure performance que celles qui ne le font pas. Cela s'explique, selon les auteurs, par le fait que les autres activités de gestion des opérations quotidiennes dépendent de la bonne prévision de la demande et de la planification globale (Riggs et Bracker, 1986:21-22).

Enfin, l'étude réalisée sur 81 épiceries a constaté que les entreprises pratiquant parallèlement la planification stratégique et la planification opérationnelle n'ont pas nécessairement une meilleure performance que celles qui utilisent la seule planification opérationnelle (Robinson, Logan et Salem, 1986:13). La même étude a relevé que les dirigeants de PME attachent plus d'importance à la planification opérationnelle qu'à la planification stratégique.

Cette dernière constatation peut se comprendre dans la mesure où certains dirigeants de PME obtiennent une bonne performance en n'utilisant que quelques activités de planification opérationnelle. Pour eux, la nécessité d'introduire la planification stratégique peut paraître douteuse. En effet, beaucoup de dirigeants de PME recherchent le profit à court terme et sont préoccupés par le contrôle direct des activités courantes : un style de gestion qui est permis par la simplicité de la structure de beaucoup de PME. Mais par ailleurs, la concurrence oblige le dirigeant à faire davantage : pour croître ou quelquefois simplement pour survivre, il doit voir plus loin. Ces exigences entraînent souvent une modification de la structure et un réajustement du style et des outils de gestion. Ce réajustement se fait généralement en partie par une meilleure délégation mais aussi par le recours à la planification stratégique. Une vision du plus long terme s'impose toujours au moment d'une réaffirmation ou d'un virage d'importance. Dans le quotidien toutefois, le dirigeant demeurera souvent principalement préoccupé et attentif aux activités de nature opérationnelle.

\subsubsection{L'utilisation de consultants en planification}

Un aspect particulier de la planification analysé par quelques-uns des auteurs pré-cités est l'utilisation de consultants en planification et son impact sur la performance.

Dans leur étude publiée en 1985, J.S. Bracker et J.N. Pearson ont constaté qu'il n'y a pas de différence significative entre le niveau de performance des entreprises utilisant des consultants dans le processus de planification et celles qui n'en utilisent pas. Cette conclusion reste vraie pour l'échantillon observé, indé-

12 Quoique certaines activités rappellent la planification à long terme, les questionnaires de l'étude se référaient clairement à des activités de court terme. 
pendamment du niveau des variables modératrices telles le niveau de sophistication de la planification, l'âge et la taille des firmes. Cette étude souligne particulièrement l'importance du rôle du propriétaire-dirigeant, plutôt que celui des consultants, dans le processus de planification, de mise en oeuvre et de contrôle du plan.

Par contre, une recherche du même type faite avant 1983 (en dehors de la période choisie pour le présent relevé) avait présenté des résultats opposés. Basée sur 101 PME de divers secteurs (usines de production, services et commerce de détail), l'étude relevait que les entreprises qui utilisent des personnes externes en planification stratégique avaient réalisé une plus forte croissance de rendement que les autres entreprises de l'échantillon (Robinson, 1982:91).

Il n'y a pas de doute que l'utilisation de consultants en planification pourrait suppléer au manque de temps et d'expérience en planification de certains dirigeants de PME. Mais elle ne peut remplacer la compétence personnelle et l'implication du dirigeant dans les activités de planification de gestion et de contrôle dans l'entreprise. De même, il faut rappeler à nouveau que la planification (avec ou sans utilisation de consultants)ne pourrait à elle seule changer le sort d'entreprises en trop sérieuses difficultés, ou modifier le niveau de performance propre à un secteur donné. Il semble donc que l'effet sur la performance de l'utilisation ou la non-utilisation de consultants en planification peut difficilement être isolé. Il ne peut être interprété indépendamment des autres variables responsables du niveau de performance dans les entreprises et secteurs d'activités compris dans les divers échantillons observés ici.

\section{Conclusions}

La présente synthèse avait pour objectif de vérifier si la planification est une réalité dans les PME et s'il y a une relation entre la planification et la performance dans ces dernières. Cette vérification s'est faite par l'analyse d'un groupe de travaux empiriques portant sur le phénomène dans des échantillons de petites et moyennes entreprises.

Déjà R.B. Robinson et J.A. Pearce avaient amorcé la réflexion sur le thème par leur compte-rendu en 1984. Rappelons leur apport.

1) La planification stratégique existe dans certaines PME, mais elle est décrite comme incomplète, non-structurée, irrégulière, sporadique, plutôt réactive et informelle. Les raisons du manque de planification dans la majorité des PME ont été analysées par ces auteurs. Ceci leur a permis d'émettre des propositions intéressantes visant la mise au point d'un processus de planification plus approprié aux PME.

2) L'effet de la planification stratégique sur la performance est un sujet de controverses. Soutenu par certaines études, l'effet positif de la planification stratégique sur la performance est remis en doute par d'autres. 
Selon les études plus récentes, l'existence de la planification stratégique dans les PME s'observe dans certaines entreprises et son effet sur la performance de celles-ci est confirmé dans une large mesure.

Toutefois, l'existence voire l'importance de la planification opérationnelle dans les PME est encore plus fréquente. Sans doute une des raisons est-elle qu'elle permet au dirigeant de mieux s'assurer le contrôle direct des opérations à court terme alors que la planification stratégique est beaucoup moins concrète et supposément moins pressante. Comme la planification stratégique doit idéalement tenir compte d'une multitude de facteurs y compris une assez grande incertitude, l'exercice est mentalement plus exigeant que celui de la planification opérationnelle; les dirigeants de PME sont généralement très peu à l'aise face à la tâche stratégique.

De façon générale, la planification stratégique apparaît liée à la performance surtout quand elle est structurée et intégrée aux orientations courantes des diverses fonctions de l'entreprise. Quant à la planification opérationnelle, son effet sur la performance est jugé meilleur quand elle est conjugée à une bonne prévision de la demande et à une planification globale dans l'entreprise. Il faut bien garder à l'esprit que ces conclusions sont essentiellement tirées de l'analyse de données empiriques, même si leur logique est également évidente.

Enfin, la conclusion la plus importante semble être que c'est moins le temps consacré à l'activité que la qualité de la planification qui détermine la performance. Bien plus, le succès est moins conditionné par l'éventail des activités de planification que par la connaissance, par le dirigeant, des principaux facteurs critiques de ses opérations. L'attention accordée à ces aspects dans la planification opérationnelle se révélerait primordiale dans l'explication d'une bonne performance. ${ }^{13}$

Au niveau pratique, il apparaît qu' une vision et une orientation à long terme sont des atouts importants pour un dirigeant de PME. Les activités spécifiques d'orientation dans les diverses fonctions de l'entreprise doivent de plus converger avec les stratégies générales de l'entreprise. Mais le dirigeant doit surtout repérer les activités opérationnelles critiques et y rester continuellement attentif. La nature de ces dernières dépend beaucoup du secteur de l'entreprise. On a pu constater toutefois que le suivi des changements du goût de la clientèle, le recensement régulier du niveau de satisfaction de cette dernière et le contrôle de la qualité, par exemple, sont des aspects souvent mis en évidence.

13 La distinction entre planification stratégique et opérationnelle est relativement claire dans certains textes; dans quelques autres, elle l'est beaucoup moins. Le lecteur devrait de plus être conscient qu'en pratique la ligne n'est pas toujours facile à tirer entre les deux types d'activités. 


\section{Remarques et recommandations}

Au niveau de la recherche, les quelques critiques suivantes semblent d'abord nécessaires :

1) Ainsi que l'ont déjà souligné R.B. Robinson et J.A. Pearce (1984), il y a un manque de continuité d'une étude à l'autre. En conséquence, très peu de travaux de recherche font suite aux interrogations laissées en suspens par ceux qui les ont précédés.

2) Peu d'études se concentrent sur un seul secteur d'activités et peu d'entre elles font le même type d'analyse sur différents secteurs à des fins de comparaison. Bien au contraire, beaucoup d'études font une analyse basée sur deséchantillons hétérogènes (prélevés dans des secteurs différents). Cela peut mettre en doute la fiabilité de leurs conclusions sur les relations entre la planification et la performance. Le niveau de performance n'est-il pas souvent beaucoup affecté par les conditions propres à chaque secteur?

3) Certaines études ne précisent pas la définition du concept de «PME». Quand elles le font, on constate que la taille des entreprises varie beaucoup d'une étude à l'autre.

4) Beaucoup d'études se préoccupent des aspects généraux de la planification ou de son formalisme, mais peu d'entre elles considèrent les aspects spécifiques du contenu des plans et de leur mise en oeuvre. Or, élaborer un plan est un chose, mais faire une bonne planification et surtout utiliser le plan comme un outil de gestion est tout autre chose.

5) Très peu d'études approfondissent le processus de planification; or, souvent le processus est bien plus important que le plan qui en découle.

6) Toutes lesétudes font appelà la participation de dirigeants d'entreprises. Il s'agit donc de répondants auto-choisis. Cela limite évidemment la représentativité de chacun des échantillons. Il est difficile de généraliser les conclusions qui en découlent. Cette contrainte en soi ne devrait pas toutefois réduire la valeur intrinsèque d'une analyse sérieuse, elle aide à mieux comprendre le phénomène. Les auteurs devraient cependant faire les mises en garde appropriées.

Plusieurs autres remarques pourraient s'ajouter aux précédentes. Au moins deux méritent certainement d'être exprimées à ce stade-ci de ce travail.

La première rappelle un débat classique. Dans quel sens se joue l'influence des variables en cause ? Les PME qui planifient réussissent mieux ? Ou Les PME qui réussissent bien planifient davantage ? Il s'agit peut-être d'une dynamique où les deux pôles sont requis. Le succès entraîne généralement le succès comme dit l'adage. Il estégalement vrai que la présence de disponibilités organisationnelles générées par le succès peut favoriser l'utilisation de méthodes de gestion plus professionnelles. La direction de la relation ne va certainement pas dans un seul sens. 
La seconde remarque a trait à la provenance de plusieurs études recensées. La lecture de ce rapport fait constater la contribution énorme de deux auteurs en tandem à la recherche sur la planification dans les petites et moyennes entreprises. R.B. Robinson et J.A. Pearce sont ceux qui, après avoir réalisé plusieurs études sur le sujet, ont publié en 1984 le premier compte-rendu général rappelé au début de cet article. Ensemble ou en collaboration avec d'autres, ils ont contribué à 5 des 12 travaux recensés ici. Chaque nouvelle publication de leur part fait un peu avancer les connaissances sur le phénomène. Il faut reconnaître le fruit de leur persistance dans une voie de recherche spécifique et oser suivre leur exemple.

Compte tenu de ces remarques, voici quelques recommandations pouvant guider de futures initiatives :

1) Il convient de faire des études secteur par secteur tenant compte de la turbulence et du dynamisme propres à chaque secteur. Peu de comparaisons valables peuvent être faites avec des groupes trop hétérogènes.

2) Etant donné que les effets de la planification prennent du temps avant de se manifester, il serait judicieux de faire des études longitudinales couvrant au moins 3 ans après l'implantation.

3) Il seraitutile de faire chaque fois une distinction entre les activités de planification stratégique et les activités de planification opérationnelle, car elles visent des horizons différents.

4) Il faudrait pouvoir décrire la nature des activités de planification stratégique et opérationnelle pour les entreprises les plus performantes, secteur par secteur. Cela permettrait de déduire clairement les configurations d'activités de planification les plus stables. C'est à partir de ces dernières qu'il serait alors possible d'élaborer les pistes d'une première théorie de la planification dans les PME. Les propositions devraient cependant intégrer déjà certaines nuances relatives aux divers niveaux possibles de turbulence dans les environnement rencontrés.

Les chercheurs ont la responsabilité de faire progresser les connaissances dans le domaine. Ils ont en particulier la tâche, de plus en plus évidente présentement, d'intégrer à leurs études deux nouvelles préoccupations. La pertinence de leur contribution en dépendra de plus en plus.

Ils doivent d'abord choisir des échantillons sectoriels pour leurs travaux. Ils pourront alors viser à identifier les facteurs critiques les plus constants dans les secteurs industriels choisis. Ainsi, ils aideront mieux le dirigeant de PME dans sa surveillance nécessaire des conditions environnementales particulières à son entreprise.

Ils doivent de plus s'efforcer de bien comprendre les exigences de l'incertitude dans le processus décisionnel du dirigeant de PME. Le dirigeant se base sur certaines hypothèses pour planifier ses opérations. Lorsque les conditions varient, ce dernier est souvent pris au dépourvu. Il est peu question de la préparation de plans de contingence par exemple dans la littérature sur la planification stratégique dans les 
PME; on retrouve rarement de plans alternatifs dans les dossiers des chefs d'entreprises. Pourtant les dirigeants de PME ont besoin de prémunir autant que possible leur firme contre les surprises à incidences stratégiques. On doit donc pouvoir développer pour eux des modèles de planification plus réalistes. Ces modèles devraient permettre une plus grande flexibilité que ceux qui leur sont généralement fournis actuellement. S'il faut changer de cap dans certaines circonstances, il faudrait être capable de le faire sans mettre la survie de l'entreprise en trop grand péril. Un processus de planification valable devrait permettre à la PME à la fois de profiter des nouvelles occasions d'affaires et de s'adapter aux circonstances menaçantes.

Le chercheur en gestion a comme première responsabilité de comprendre la réalité des entreprises. Beaucoup reste à faire sur le sujet de la planification stratégique dans les petites et moyennes entreprises. Le chercheur dans ce domaine a également la responsabilité évidente de proposer de nouveaux modèles de gestion plus adéquats. Les dirigeants de PME les réclament pour l'orientation stratégique de leurs entreprises. N'hésitons pas à les proposer.

\section{BIBLIOGRAPHIE}

Ackelsberg, R. et Arlow, P., (1985), «Small business do plan and it pays off», Long Range Planning, vol. 18, $\mathrm{n}^{\circ}$ 5, p. 61-67.

Bracker, J.S., (1982), «Planning and financial performance among small entrepreneurial firms: an industry study», Unpublished Doctoral Dissertation, Georgia State University.

Bracker, J.S. et Pearson, J.N., (1985), «The impact of consultants on small firm strategic planning», Journal of Small Business Management, vol. 23, $\mathrm{n}^{\circ} 3, \mathrm{p} .23-30$.

Bracker, J.S. et Pearson, J.N., (1986), «Planning and financial performance of small mature firms», Strategic Management Journal, vol. 7, ${ }^{\circ} 6$, p. 503-522.

Bracker, J.S., Keats, B.W. et Pearson, J.N., (1988), «Planning and financial performance among small firms in growth industry», Strategic Management Journal, vol. 9, $\mathrm{n}^{\circ}$ 6, p. 591-603.

Chakravarthy, B.S., (1987), «On tailoring a strategic planning system to its context: some empirical evidence», Strategic Management Journal, vol. 8, n 6, p. 517-534.

Chenhall, R.H. et Romano, C.A., (1989), «Formal planning and control presence and impact on the growth of small manufacturing firms», communication présentée au $34 \mathrm{e}$ congrès mondial du International Council for Small Business, Québec, juin.

Chicha, J.K. et Julien, P.A., (1979), «La stratégie des PME et leur adaptation au changement», Rapport de recherche, Université du Québec à Trois-Rivières, Trois-Rivières (Québec).

d'Amboise, G., (1989), La PME canadienne, situation et défis, Institut de Recherches Politiques, Presses de l'Université Laval, Québec.

Dilts, J.C. et Prough, G.E., (1989), «Strategic options for environmental management: a comparative study of small vs large entreprises», Journal of Small Business Management, vol. 27, $\mathrm{n}^{\circ} 3$, p. 31-38.

Gable, M.et Topol, M.T.,(1987), «Planning practices of small scale retailers», AmericanJournal of Small Business, vol. 12, $\mathrm{n}^{\circ} 2$, p. 12-32. 
Gasse, Y., (1979), «Management techniques and practices in small manufacturing firms», Comptes rendus de International Council for Small Business, Québec.

Hartman, S. et Lundberg, D., (1989), «The role of planning in small vs large businesses: do managers report preferences for different tools ?», Comptes rendus du 34e congrès mondial de International Council for Small Business, Québec, juin, p. 500-517.

Julien, P.A. et Marchesnay, M., (1988), «La place de la petite entreprise dans les économies en mutation», dans La petite entreprise, principes d'économie et de gestion, Librairie Vuibert, Paris, p. 39-49.

King, W.R., (1983), «Evaluating strategic planning systems», Strategic Management Journal, vol. $4, n^{\circ} 4$, p. 263-277.

Mayer, K. et Goldstein, S., (1961), «The first two years: problems of small firms growth and survival», Small Business Administration, Washington, D.C.

Najjar, M., (1966), «Planning in small manufacturing firms», Unpublished Doctoral Dissertation, Ohio State University.

Orpen, C., (1985), «The effects of long range planning on small business performance: A further examination», Journal of Small Business Management, vol. 23, $\mathrm{n}^{\circ} 1, \mathrm{p} .17-23$.

Pearce, J.A., Freeman, E.B. et Robinson, R.B., (1987), «The tenuous link between formal strategic planning and financial performance», Academy of Management Review, vol. 12, $\mathrm{n}^{\circ} 4$, p. 658-675.

Pearce, J.A., Robinson, R.B. et Robbins, D.K., (1987), «The impact of grand strategy and planning formality on financial performance», Strategic Management Journal, vol. 8, $\mathrm{n}^{\circ} 1$, p. 125-134.

Ramanujam, V.et Venkatraman, N., (1987), «Planning and performance: a new look on an old question», Business Horizons, , vol. 30, $\mathrm{n}^{\circ} 3$, p. 19-25.

Riggs, W.E. et Bracker, J.S., (1986), «Operations management and financial performance», American Journal of Small Business, vol. 10, $\mathrm{n}^{\circ} 3, \mathrm{p} .17-23$.

Robinson, R.B., (1979), «Forecasting and small business: a study of the strategic planning process», Journa! of Small Business Management, vol. 17, $\mathrm{n}^{\circ}$ 3, p. 19-27.

Robinson, R.B., (1980), «An empirical investigation of the impact of SBDC - Strategic planning consultation upon the effectiveness of small business in Georgia», Unpublished Doctoral Dissertation, University of Georgia.

Robinson, R.B., ( 1982), «The importance of outsiders in small firm strategic planning», Academy of Management Journal, vol. 25, $\mathrm{n}^{\circ} 1, \mathrm{p}$ 80-93.

Robinson, R.B., (1983), «The impact of formalized strategic planning on financial performance in small organizations», Strategic Management Journal, vol. 4, $n^{\circ} 3$, p. 197-207.

Robinson, R.B. et Pearce, J.A., (1984), «Research thrusts in small firm strategic planning», Academy of Management Review, vol. 9, $\mathrm{n}^{\circ} 1$, p. 128-137.

Robinson, R.B., Pearce, J.A., Vozikis, G.S. et Mescon, T.S., (1984), «The relationship between stage of development and small firm planning and performance», Journal of Small Business Management, vol. 22, $\mathrm{n}^{\circ} 2$, p. 45-52.

Robinson, R.B., Logan, J.E. et Salem, M.Y., (1986), «Strategic versus operational planning in small retail firms", American Journal of Small Business, vol. 10, n 3, p. 7-16.

Robinson, R.B., Salem, M.Y., Logan, J.E. et Pearce, J.N., (1986), «Planning activities related to independent retail firm performance», American Journal of Small Business, vol. 11, $\mathrm{n}^{\circ} 1, \mathrm{p} .19-26$.

Sexton T.M. et Dable, R.D., (1976), «Factors affecting long-range planning in the small business firm», Marquette Business Review, vol. 20, n 4, p. 158-165. 
Sexton, D.L. et Van Auken, P., (1985), «A longitudinal study of small business strategic planning», Journal of Small Business Management, vol. 23, $\mathrm{n}^{\circ} 1, \mathrm{p} .7-15$.

Shuman, J.C., (1975), «Corporate planning in small companies», Long RangeP lanning, vol. 8, $\mathrm{n}^{\circ}$ 5, p. 81-90.

Smith, N., (1967), The Entrepreneur and his Firm: The Relationship Between Type of Man and Type of Company, Michigan State University, East Lansing, MI.

Sova, P., (1989), «The business plan and planning in small business», Communication présentée au 34e congrès mondial du International Council for Small Business, Québec, juin.

Steiner, G., (1979), Strategic Planning, New York, The Free Press.

Still, T.W., (1974), «An exploratory investigation of strategic planning behavior in small firms», Unpublished Doctoral Dissertation, Florida State University

Trow, D.B., (1961), «Executive succession in small compagnies», Administrative Science Quarterly, vol. 25, $\mathrm{n}^{\circ} 1$, p. 12-15.

Wyant, R., (1977), «The business failure record», New York, Dun and Bradstreet. 